\title{
Chronic endometritis: an hidden pathology
}

Keywords: CD138, endometrium, hysteroscopy, chronic endometritis

\section{Introduction}

Chronic endometritis is a chronic inflammatory condition of the endometrium characterized by the presence of plasma cells in the endometrial stroma. ${ }^{1,2}$ The alterations of the endometrial microenvironment can alter the production of endometrial cytokines, thus damaging endometrial function and leading to abnormal lymphocyte patterns in the endometrium. Together with altered secretion of paracrine factors, can reduce the receptivity of embryos causing female infertility. ${ }^{3-5}$

In $10-11 \%$ of women who undergo a "benign cause" hysterectomy ${ }^{6}$ In $33.3-57.55 \%$ patients with infertility chronic endometritis is present. This is especially frequent in cases of repeated abortions and recurrent implantation failure after in vitro fertilization (IVF). ${ }^{1,7}$

Infection appears to be the basis of chronic endometritis. Some pathogen is found in up to $73.1 \%$ of patients ${ }^{6}$.Common bacteria and Mycoplasma are the most frequently pathogens in chronic endometritis. Although female genital tuberculosis can cause chronic endometritis and infertility, it is anecdotal in industrialized countries and frequently is associated with predisposing factors. ${ }^{5,6}$ The endometrial cultures were negative in about $31.7 \%$ of the cases, probably due to the presence of nocultivable pathogen (like anaerobic bacterias or viruses) Different studies have shown that antibiotic therapy can restore normal endometrial histology, improving the rate of implantation.

\section{Diagnosis}

Diagnosis is a great challenge. It is frequently under diagnosed, cause of clinical manifestations, ultrasound and analytical markers are unspecified. ${ }^{1,3,8,9}$

While some patients are asymptomatic, others have dysfunctional uterine bleeding, pelvic algae, dyspareunia, and leucorrhea. ${ }^{1,3}$ The most common symptoms is vaginal bleeding (reported in up to $94 \%$ of the patients).${ }^{10}$ Blood markers of infection, such as leukocytosis or C-reactive protein, do not appear elevated. ${ }^{5}$

Focal or diffuse periglandular hyperemia, increased thickness of the endometrium that appears white and with an irregular surface, micro-polyps (less than $1 \mathrm{~mm}$ in size), pedunculated stromal edema are some of the diagnostic hysteroscopy signs. These lesions can be diffuse or cover most of the endometrial surface. ${ }^{10}$ The relationship between endometrial polyps and chronic endometritis is unclear. In a recent study, an association between vascular changes in the functional layer of the endometrium and signs of chronic endometritis has been found, supporting the hypothesis that the vascular axis of the endometrial polyp represents an evolutionary stage of vasculopathy due to chronic endometritis (Figure 1) (Figure 2). ${ }^{2}$

While sensitivity of hysteroscopy is $98.4 \%$, its specificity is $56.23 \%$, the positive predictive value is $63.5 \%$, and its negative predictive value is $97.52 \% .^{11}$ For this reason, hysteroscopy is especially useful in diagnosis recognizing specific signs of chronic endometritis. ${ }^{1,11,12}$ In these patients, two samples should be taken, one for a microbiological study and the other for a histopathological study. Cicinelli et al have obtained in their studies a diagnostic correlation
Volume II Issue 3 - 2020

\author{
Vendrell Aranda Celia María,' Duro Gómez \\ Jorge ${ }^{1,2}$ \\ 'Reina Sofía University Hospital, Córdoba, Spain \\ ${ }^{2}$ San Juan de Dios Hospital. Córdoba, Spain
}

Correspondence: Duro Gómez Jorge, Reina Sofía University Hospital, San Juan de Dios Hospital, Calle Arabista Joaquina Eguaras $\mathrm{N}^{\circ} 2$ Esc 4 I ${ }^{\circ} \mathrm{B}$ Córdoba CP I40II, Spain,

Tel +34685810803, Email Jorgedurogomez@gmail.com

Received: April 14, 2020 | Published: May 15, 2020

of up to $93.4 \%$ based on hysteroscopic criteria during the follicular phase that suggest chronic endometritis. ${ }^{2,7}$

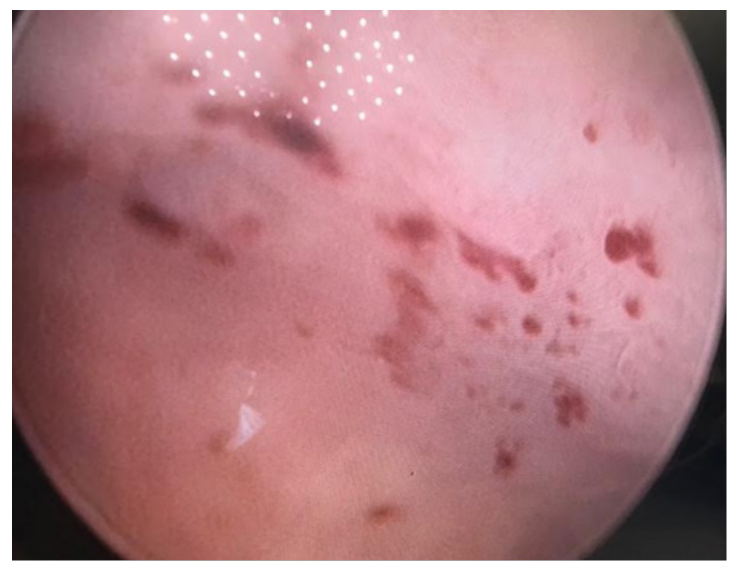

Figure I Tipical periglandular hyperhemia.

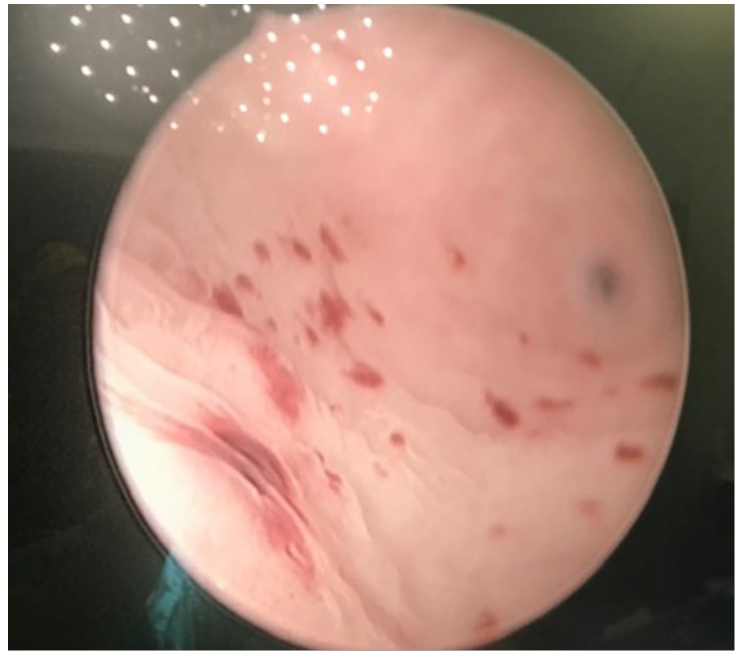

Figure 2 Tipical periglandular hyperhemia and irregular endometrial surface. 
Bacterias are the most present infectious agent. It is possible found Streptococcus $(27 \%)$, E. coli $(11 \%)$, Enterococcus faecalis $(14 \%)$ and Urea plasmaurealyticum $(11 \%))^{1,9}$ The presence of Chlamydia trachomatis is only $2.7 \%$, and that of Neisseria gonorrhoeae is practically undetectable as a cause in chronic endometritis. ${ }^{4,6}$

From a histological point of view, it is common to find leukocytes in the endometrial lining, especially in the phase prior to menstruation. To increase diagnostic precision, immune histochemistry is recommended, in addition to conventional histology. ${ }^{8}$ Hematoxylin and eosin study is less sensitive (13\%) than the CD138 immuno histochemical study $(56 \%)^{6}$ to detect plasma cells in the stroma. endometrial. Syndecan 1, also known as CD138, is a transmembrane proteoglycan that is found on the surface of plasma cells and keratinocytes, but not on mononuclear cells, lymphocytes, or endometrial stromal cells. For this reason, its detection is used for diagnosis of chronic endometritis. ${ }^{2,5,8,9}$

Regarding the complementary ultrasonograpy, transvaginalsonography have no specific sign but free pelvic fluid on ultrasound appears to be associated with positive CD138 on endometrial collection. ${ }^{8}$

\section{Treatement}

Doxycycline $100 \mathrm{mg}$ each 12 hours for 14 days removes plasma cells CD138 from $70 \%$ to $96 \%$ of histological samples. ${ }^{5,6}$

Some studies recommends treat it according to the bacterial sample:

In Gram negative bacteria ciprofloxacin 500mg is used twice a day for 10 days. In the case of Gram positive bacteria amoxicillin 1 gram plus clavulanic acid was prescribed twice a day for 8 days.

In case of negative bacterial sample but histological diagnosis of chronic endometritis, a treatment based on Ceftriaxone $250 \mathrm{mg}$ IM administered in a single dose together with Doxycycline $100 \mathrm{mg}$ orally twice a day for 14 days and Metronidazole 500mg orally twice a day for 14 days could be useful, according to Centers for Disease Control guidelines.

In case of persistent signs of chronic endometritis in subsequent hysteroscopy, the protocol could be repeated up to three times. 5,7

Antibiotic treatment can attenuate the effect of chronic endometritis on infertility: in the study by Cicinelli et al, the pregnancy rate of the group with normalization of hysteroscopy after antibiotics was significantly higher than that of the group that did not respond to antibiotic therapy $(61 \%$ vs. $10 \%)$. Moreover, McQueen et al study shows that the rate of live births per pregnancy in patients with chronic endometritis increased significantly to $56 \%$ after antibiotic treatment, compared to $7 \%$ before of treatment.

Endometritis does not resolve in all cases after antibiotherapy. Several studies propose an hysteroscopy verification after antibiotherapy, modifying the treatment according to the clinical, hysteroscopy and histological findings. $., 5,7$

\section{Acknowledgments}

None.

\section{Funding}

None.

\section{Conflicts of interest}

All authors declare that they have no competing interests.

\section{References}

1. Cicinelli E, Matteo M, Trojano G, et al. Chronic endometritis in patients with unexplained infertility: Prevalence and effects of antibiotic treatment on spontaneous conception. Am J ReprodImmunol. 2018;79(1):e12782.

2. Cicinelli E, Bettocchi S, de Ziegler D, et al. Chronic endometritis, a common disease hidden behind endometrial polyps in premenopausal women: first evidence from a case-control study. J Minim Invasive Gynecol. 2019;26(7):1346-1350.

3. VItagliano A, Saccardi C, Noventa M, et al. Effects of chronic endometritis therapy on in vitro fertilization outcome in women with repeated implantation failure: a systematic review and meta-analysis. Fertil Steril. 2018;110(1):103-112.

4. Cicinelli E, Matteo M, Tinelli R, et al. Chronic endometritis due to common bacteria is prevalent in women with recurrent miscarriage as confirmed by improved pregnancy outcome after antibiotic treatment. Reprod Sci. 2014;21(5):640-647.

5. Puente E, Alonso L, Lagana AJ, et al. Chronic endometritis: old problem, novel insights and future challenges. Int J FertilSteril. 2020;13(4):250256.

6. Park HJ, Kim YS, Yoon TK, et al. Chronic endometritis and infertility. Clin Exp Reprod Med. 2016;43(4):185-192.

7. Cicinelli E, Matteo M, Tinelli R, et al. Prevalence of chronic endometritis in repeated unexplained implantation failure and the IVF success rate after antibiotic therapy. Hum Reprod. 2015;30(2):323-330.

8. Fan X, Li X, Li Y, et al. Endometrial CD138 count appears to be a negative prognostic indicator for patients who have experienced previous embryo transfer failure. Fertil Steril. 2019;112(6):1103-1111.

9. Li Y, Yu S, Huang C, et al. Evaluation of peripheral and uterine immune status of chronic endometritis in patients with recurrent reproductive failure. Fertil Steril. 2020;113(1):187-196.

10. Cicinelli E, Tinelli R, Lepera A, et al. Correspondence between hysteroscopic and histologic findings in women with chronic endometritis. Acta Obstet Gynecol Scand. 2010;89(8):1061-1065.

11. Zolghadri J, Momtahan M, Aminian K, et al. The value of hysteroscopy in diagnosis of chronic endometritis in patients with unexplained recurrent spontaneous abortion. Eur J Obstet Gynecol. 2011;155(2):217-220.

12. Parry JP, Isaacson KB. Hysteroscopy and why macroscopic uterine factor matter for fertility. Fertil Steril. 2019;112(2):0015-0282. 